\title{
Knee Joint Distraction in a Dog as Treatment for Severe Osteoarthritis
}

\author{
Michelle Teunissen ${ }^{1} \quad$ Simon C. Mastbergen ${ }^{2}$ Dennis C. Spoelman ${ }^{3} \quad$ Floris P. Lafeber $^{2} \quad$ Irene S. Ludwig ${ }^{4}$ \\ Femke Broere $^{1,4}$ Marianna A. Tryfonidou ${ }^{1}$ Björn P. Meij ${ }^{1}$
}

\footnotetext{
1 Department of Clinical Sciences, Faculty of Veterinary Medicine, Utrecht University, Utrecht, the Netherlands

2 Department of Rheumatology and Clinical Immunology, UMC Utrecht, Utrecht, the Netherlands

${ }^{3}$ Department of Clinical Sciences, Dierenartspraktijk IJ van Rijn, Nieuw-Vennep, the Netherlands

${ }^{4}$ Department of Biomolecular Health Sciences, Faculty of Veterinary Medicine, Utrecht University, Utrecht, the Netherlands
}

Address for correspondence Michelle Teunissen, MSc, DVM, Department of Clinical Sciences, Faculty of Veterinary Medicine, Utrecht University, Yalelaan 1083584 CM, Utrecht, The Netherlands (e-mail: m.teunissen@uu.nl).

\begin{abstract}
Keywords

- dog

- knee joint distraction

- osteoarthritis

- force plate

- synovial fluid biomarkers

Osteoarthritis (OA) is the most common joint disease diagnosed in veterinary practice. There is no cure; where conservative treatment fails, a joint prosthesis is the last resort. In human OA patients, temporary distraction is a joint-preserving treatment which provides clinical and structural benefits, and postpones the need for total knee replacement for up to 9 years. In this single case, feasibility of knee joint distraction (KJD) was assessed in a 9-year-old female Dobermann with severe OA. Osteoarthritis of the left stifle joint was diagnosed clinically and radiographically. Knee joint distraction was applied for 7 weeks using a custom-made, hinged, external fixator. Follow-up of 1 year included owner questionnaires, orthopaedic examination, radiography, and force plate analysis. Additionally, synovial fluid biomarkers were assessed. Application and removal of the KJD frame were successfully accomplished without surgical complications. During KJD, the dog tolerated the frame well and maintained the use of the left hindlimb. Mild pin tract infection developed during the distraction period, which responded well to oral antibiotic medications. Owners reported clinical improvement during $\mathrm{KJD}$ and the follow-up period. This was confirmed by force plate analysis, demonstrating improvement from 3 months and onward. KJD proved to be a feasible strategy to treat end-stage $\mathrm{OA}$ in this single case; clinical efficacy remains to be determined in a larger cohort.
\end{abstract}

\section{Introduction}

Osteoarthritis (OA), the primary joint disease in dogs, is characterized by cartilage degradation, subchondral bone changes and synovitis. ${ }^{1}$ Clinical signs include progressive reduced limb function, stiffness and behavioural changes, resulting in a reduced quality of life. Treatment of OA is mostly conservative, consisting of pain management com-

received

June 6, 2021

accepted after revision

November 16, 2021
DOI https://doi.org/

$10.1055 / \mathrm{s}-0042-1742471$.

ISSN 2625-2325. bined with nutritional, weight and exercise management. Surgical treatment includes joint debridement, arthrodesis or total joint replacement. The latter remains a challenging procedure in end-stage $\mathrm{OA}$, and is, so far, only performed on a routine basis in the hip joint. ${ }^{2,3}$

In humans, joint distraction is used to treat severe $\mathrm{OA}$ and delays the need for joint replacement for several years. ${ }^{4}$ During

(c) 2022. The Author(s).

This is an open access article published by Thieme under the terms of the Creative Commons Attribution License, permitting unrestricted use, distribution, and reproduction so long as the original work is properly cited. (https://creativecommons.org/licenses/by/4.0/)

Georg Thieme Verlag KG, Rüdigerstraße 14, 70469 Stuttgart, Germany 
joint distraction, the two bony ends of a joint are temporarily separated using an external fixation frame. Currently, joint distraction is mostly applied for knee $\mathrm{OA},{ }^{4,5}$ though other joints, such as the ankle, can be treated as well. ${ }^{6,7}$ Human clinical studies report that knee joint distraction (KJD) resulted in clinical, radiographic and MRI improvement, with evidence for tissue repair at 1 and 2 years after $\mathrm{KJD},{ }^{8-10}$ and clinical improvement reported for at least 9 years. ${ }^{5,11}$ In experimental canine $O A,{ }^{12}$ structural improvement was seen 25 weeks after a 8 -week KJD treatment. ${ }^{13}$ This improvement consisted of improved macroscopic and histopathologic cartilage scores, higher proteoglycan content, better retention of newly formed proteoglycans and less collagen damage, compared with the untreated OA knee joints. $^{13}$

The mechanism behind distraction is not exactly known. The combination of decreased mechanical joint loading and the intermittent fluid pressure changes, caused by the springs or hinges in the frame, is thought to create a supportive environment that initiates cartilage repair activity. ${ }^{13,14}$

The objective of this case report was to assess whether $\mathrm{KJD}$ is a feasible alternative treatment option for end-stage canine stifle OA with regard to safety and owner experience. For this purpose, KJD was applied to a dog with severe, chronic stifle OA without joint instability or other underlying joint or bone diseases.

\section{Case Report}

\section{History and Clinical Examination}

A 9-year-old, $40 \mathrm{~kg}$, neutered female Dobermann was presented with a history of intermittent left hindlimb lameness (with periods of non-weight bearing), difficulty rising and an overall decreased quality of life as perceived by the owner (restlessness at night, decreased activity). Attempted weight management, and the oral administration of carprofen and tramadol, failed to improve the dogs' quality of life. On clinical examination, a $2 / 4^{15}$ left hindlimb lameness was present. Both stifles were thickened, with evident joint effusion and crepitation. Passive flexion and extension of the left hindlimb evoked pain, with a negative drawer sign and tibial compression test, and absence of pain from the medial meniscal compartment. Radiography revealed bilateral severe chronic degenerative stifle OA with moderate joint effusion and periarticular soft tissue swelling (Morgan OA score ${ }^{16}$ left: 25 , right: 14) (-Fig. 1). Altogether, chronic degenerative OA without evidence for stifle joint instability was diagnosed. The dog was an appropriate candidate for KJD due to her size (i.e. fitted the custom-made frame, adapted to the canine stifle anatomy and used in the previous experimental studies ${ }^{13}$ ) and the absence of concurrent joint and bone pathologies. The left stifle joint was chosen for KJD treatment as it was clinically more severe.

Owner consent for KJD and subsequent follow-up was obtained. In addition, ethical approval (no. AVD1080020173964) was obtained for the collection of synovial fluid (SF) for research purposes.

\section{Surgical Distraction Procedure}

Surgery was performed under general anaesthesia according to standard of care procedures and pre-emptive antibiotic medications (cefazolin). A femoral-sciatic nerve block, using levobupivacaine, was placed preoperatively, and perioperative pain management was provided with carprofen and buprenorphine. The negative drawer and tibial compression test were confirmed under sedation.

The stainless-steel bone pins (Stryker, Stryker Nederland BV) were negatively threaded, $4 \mathrm{~mm}$ diameter $(\varnothing)$ (150 mm length) or $\emptyset 3 \mathrm{~mm}$ (110 $\mathrm{mm}$ length), and were drilled manually and percutaneously under fluoroscopic guidance, using a C-arm, to ascertain that pins were parallel to each other and engaged the trans cortex ( - Fig. 2, Supplementary Material). The bone pins were connected to the external fixation frame $(\varnothing 5 \mathrm{~mm})$ using commercially available connectors (Delta coupling, Stryker). The femoral and tibial parts of the frame were connected medially and laterally of the stifle joint by a construction that consists of two threaded rods with a hinge in between ( - Fig. 2A, yellow arrow). Finally, the joint was distracted by extending the connecting rods for approximately $5 \mathrm{~mm}$ ( -Fig. 2B, blue arrow). Smooth motion of the joint during
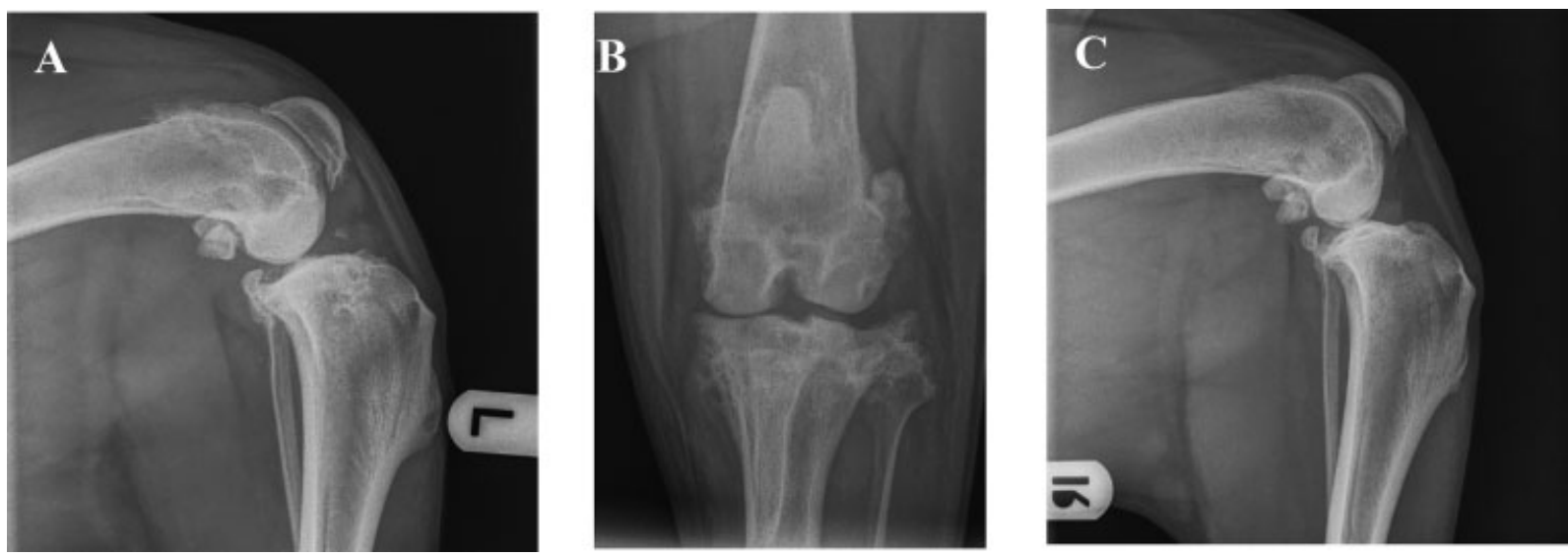

Fig. 1 Mediolateral (A) and caudocranial (B) radiographic images of the left stifle joint and right stifle joint (C) prior to referral. The left stifle joint of the 9-year-old Dobermann showed signs of degenerative joint disease with moderate joint effusion and synovial proliferation. In addition, marked peri-articular osteophytes $(>5 \mathrm{~mm}$ ) were present on the lateral and medial margins of the tibial plateau, medial and lateral epicondyles, fabellae, the apex of the patella and the distal aspect of the femoral trochlea. 

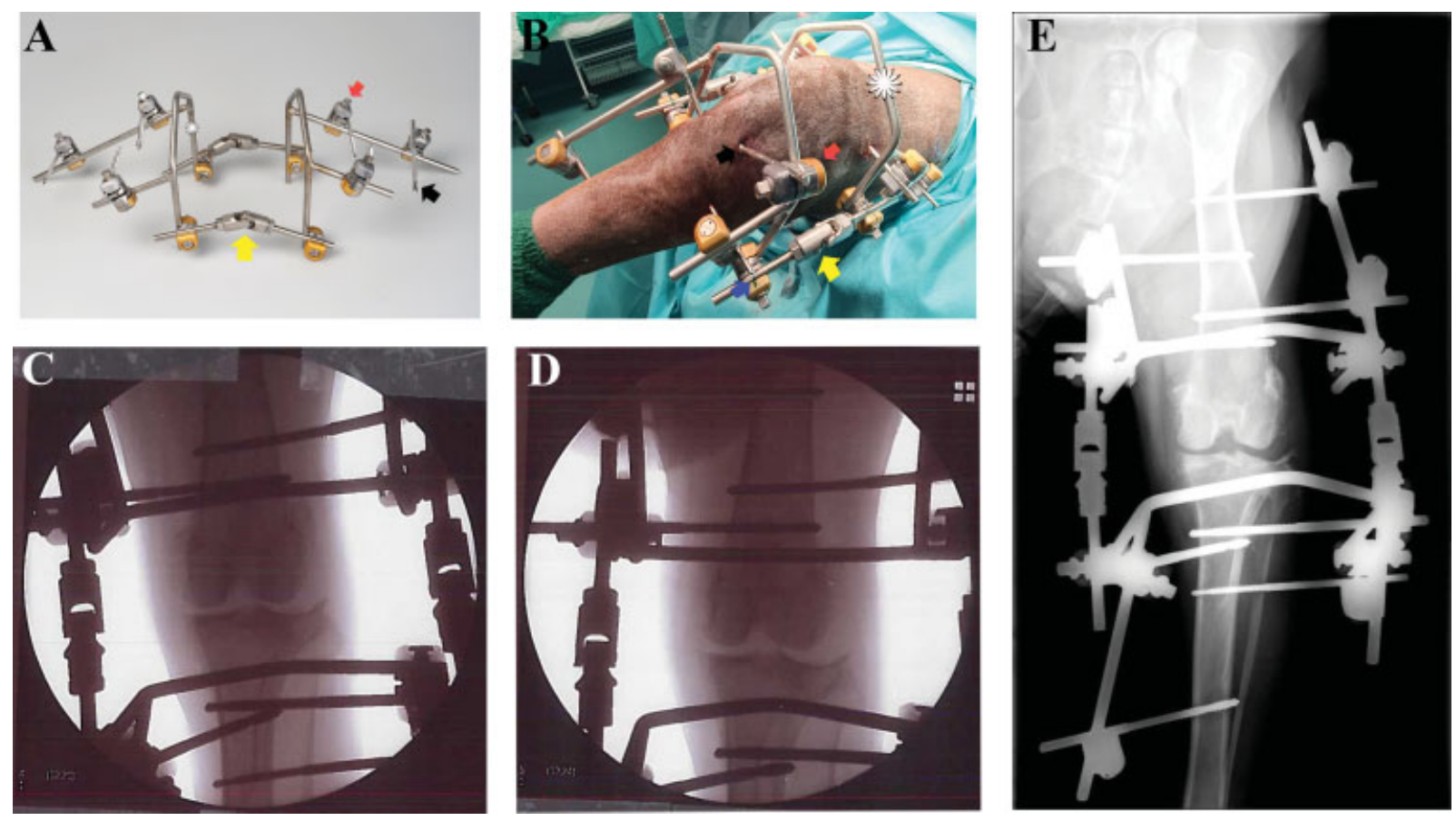

Fig. 2 Placement of the distraction frame. (A) Distraction frame consisting of the (B) bone pins (black arrow point) that were drilled into the femur and tibia of the left hindlimb. An external fixation frame (white star), custom-made and adapted to the canine knee anatomy, was connected to the bone pins with connectors (red arrow point). Furthermore, the external frames of the femur and tibia were connected medially and laterally by extendable hinges (yellow arrow point). (C) Placement of the bone pins was visualized during surgery by fluoroscopy using a Carm. (D) Distraction of the joint was achieved by extending the hinges (blue arrow) and was verified by fluoroscopy. (E) Postoperative radiograph.

flexion and extension was maintained, achieving a range of motion of approximately 60 degrees.

The patient was discharged on the same day with oral carprofen, 7 days of oral amoxicillin clavulanic acid (Synulox; $12.5 \mathrm{mg} / \mathrm{kg}$; Zoetis BV; based on the protocol of experimental studies $^{13}$ ) and recommendation of regular but light exercise with emphasis on weight bearing of the treated left hindlimb. The owner reported weight bearing on the left hindlimb at day 1 after surgery.

At 9 days after surgery, the patient was presented with sudden 2-3/4 lameness of the treated limb without a specific cause. On clinical examination, the medial distal femoral pin tract was found to be infected. The owner was recommended to clean and disinfect the pin tracts daily and oral antibiotic treatment was resumed until the end of the KJD treatment. No other soft-tissue tension problems were observed during the study.

At week 2, 4, and 6 of KJD, clinical evaluations were scheduled with special attention to the pin tracts (-Supplementary Fig. S1). Additionally, radiographs were taken to assess the integrity of the bone pins and to confirm KJD. At 4 weeks, the distracted joint width was judged to be suboptimal, in particular a collapse of the medial part of the lateral condyle was observed. This was adjusted by adding $3 \mathrm{~mm}$ of distraction on the connecting rods on both sides of the stifle joint (-Supplementary Fig. S2).

After 7 weeks of KJD, the frame and bone pins were removed under general anaesthesia. A small fissure was found in one of the medial rods of the femoral fixation frame, which upon manipulation extended to a full crack. Retro- spective evaluation of the radiographs showed that a very small fissure in the rod was visible after 6 weeks of KJD. None of the bone pins failed during KJD.

After KJD treatment, the owner was recommended to build up exercise slowly, starting with light, leashed exercise (10-15 minutes, 4-6 times a day). Within 4 weeks after KJD, the owner reported that the patient was walking better than before the treatment and was more active and playful, making it difficult to restrain her. At 4 weeks after KJD, clinical evaluation showed full recovery of the pin tract wounds, while proliferation of the cortical bone surrounding the pin tracts was still palpable. A 1/4 lameness was still present, but the dog was noticeable more active. After this evaluation, the exercise regime was increased to normal routine and the carprofen dosage was lowered.

At 2 months after KJD, the owner reported persistent clinical improvement. On clinical examination, no lameness was detected, and oral analgesia was halted. At the end of the follow-up, that is, at 12 months after KJD, clinical improvement was still present and the dog was not receiving pain medication. Radiographic evaluation at 1-, 6- and 12-month follow-up showed no OA progression of the left stifle (more details in (-Supplementary Fig. $\mathbf{5 3}$ ).

\section{Questionnaires}

The owners were initially concerned about the interference of the frame with the mobility of their dog. However, they reported that the dog adapted relatively fast, with improved mobility compared with baseline. At the end of the study, the owners were extremely content with the results. Pain and 

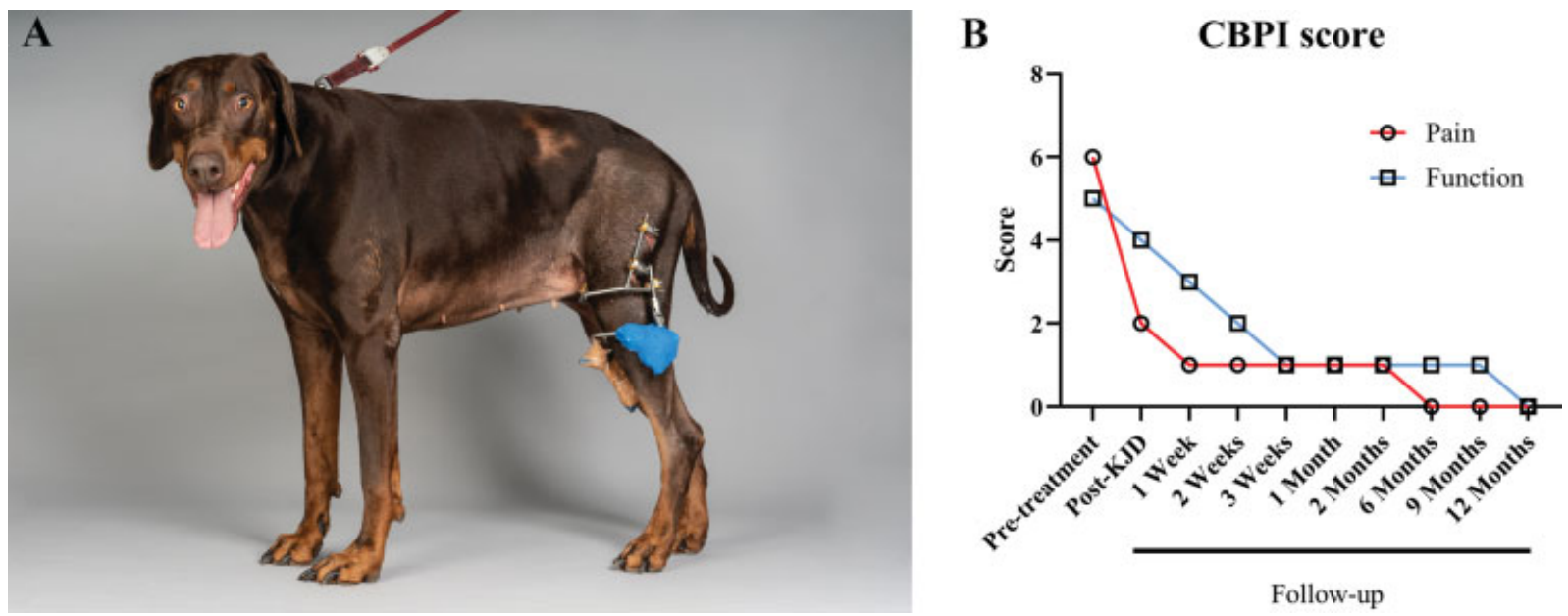

Fig. 3 Post-surgical monitoring. (A) Every 2 weeks a clinical examination was performed of the patient with special attention to the pin tracts. Pin-tract infection of the femoral bone pins was present and caused ulceration of the skin surrounding the bone pins. (B) The Canine Brief Pain Inventory (CBPI) consists of two parameters; the description of pain severity ('Pain': red line) and the description of the interference with function ('Function': blue line). The mean of the multiple parameter scores of each parameter is provided. The CBPI was completed by the owner before treatment (pre-treatment), directly after treatment with joint distraction (post-knee joint distraction [KJD]) and in the follow-up period.

functionality were assessed using the Canine Brief Pain Inventory (CBPI $)^{17}$ at baseline, directly after $\mathrm{KJD}$, at 1,2 , and 3 weeks, and $1,2,6,9$, and 12 months of follow-up (-Fig. 3). At baseline, the CBPI pain score was 6/10. The average functionality score at baseline was a 5/10. Additionally, the owner rated the quality of life as poor. Directly after $\mathrm{KJD}$, a reduced pain inventory score of $2 / 10$ was reported, which decreased further to $1 / 10$ at two weeks after KJD. From 6 months and onwards, up until the end of the study, a pain score of 0/10 was reported by the owner with an excellent quality of life.

\section{Force Plate Analysis}

Force plate analysis was performed as described by Suwankong and colleagues. ${ }^{18}$ Briefly, vertical (Fz) and craniocaudal (Fy) forces at walking speed were measured using a force plate mounted flush with the surface of an 11-m walkway at a sampling frequency of $100 \mathrm{~Hz}$. At baseline, the peak vertical force (PVF) was comparable in both hindlimbs, with a decreased pelvic/thoracic ratio $(\mathrm{P} / \mathrm{T}$ ratio) compared with the reference $^{18}$ (-Fig. 4). The peak breaking force and propulsive force (PPF) were lower in the left compared with the right hindlimb, and their respective $\mathrm{P} / \mathrm{T}$ ratios were decreased compared with the reference value. ${ }^{18}$ One month after KJD, the PVF and PPF of the distracted left hindlimb and the $\mathrm{P} / \mathrm{T}$ ratios were all decreased compared with baseline (-Fig. 4). From 6 months and onwards, the PVF increased in the distracted left hindlimb compared with baseline. Additionally, at this time point, the $\mathrm{P} / \mathrm{T}$ ratio of the PVF increased to the level of the reference values. Although the PPF never reached the baseline level, the P/T ratio reached the reference value at 6 months after follow-up to decrease again at 1 year.

\section{Synovial Fluid Analysis}

Synovial fluid (SF) samples were obtained at baseline (before surgery), directly after KJD and at 6 months of follow-up. At 9 and 12 months of follow-up, SF collection was unsuccessful, due to the low volume of SF. After collection, SF was kept on ice, centrifuged ( 5 minutes, $500 \mathrm{~g}, 4^{\circ} \mathrm{C}$ ) to remove cellular debris, aliquoted and stored at $-80^{\circ} \mathrm{C}$ until further analysis. Synovial fluid samples were pre-treated with hyaluronidase $(4 \mathrm{mg} / \mathrm{mL}$, Sigma) for 15 minutes at $37^{\circ} \mathrm{C}$ and analysed using a canine multiplex ELISA (MILLIPLEX, CCYTOMAG-90K, Merck KGaA, Darmstadt, Germany) according to the manufacturer's protocol and a Luminex analyser (MAGPIX, Luminex B.V., 'sHertogenbosch, the Netherlands). Interleukin(IL)-6 and IL-8 SF levels decreased directly after KJD and even further at 6 months follow-up. Interleukin-7 and -15 were detected only directly after KJD, while CCL2 was decreased directly after KJD (-Fig. 5). Interferon- $\gamma$, IL-2, -10, and -18 and tumour necrosis factor- $\alpha$ were not detected.

\section{Discussion}

With the exception of the hip joint, where large cohorts have been published, ${ }^{19}$ total stifle and elbow replacements in dogs remain in the preclinical phase. ${ }^{2,20}$ As such, joint-preserving treatment strategies for severe canine OA are needed. Joint distraction elicits prolonged clinical and structural benefits in human clinical trials. The present case demonstrates the feasibility of KJD in a canine patient with severe, radiographically confirmed, OA.

Knee joint distraction is an invasive treatment that requires the intensive postoperative care that comes with external fixators. ${ }^{21}$ Nonetheless, the owner perceived this treatment as acceptable. After a short adjustment period for both the owners and the dog, the dog accepted the KJD treatment with minimal problems.

One important complication of KJD was the presence of pin tract infections, a complication also reported in human patients, ${ }^{4,14}$ and in veterinary practise using external skeletal fixation for fracture repair. ${ }^{22,23}$ Although pin tract infections represent a substantial burden to the patient, they can be 
A

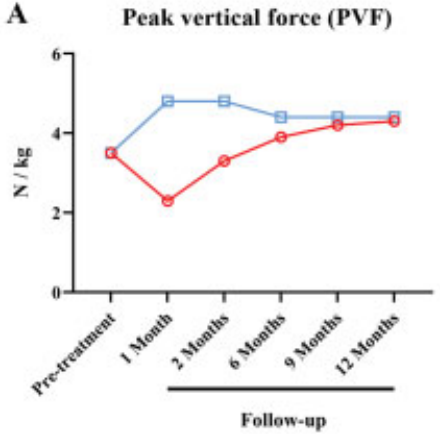

D

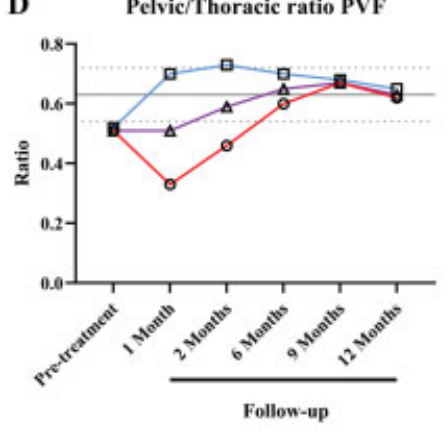

B

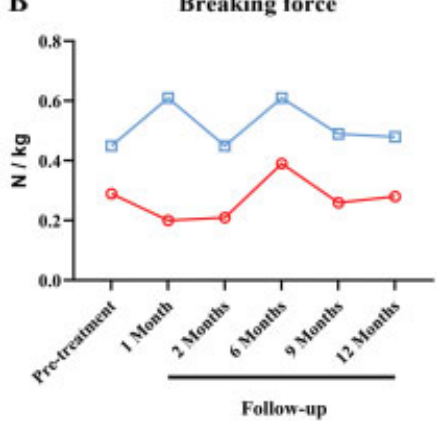

$\mathbf{E}$

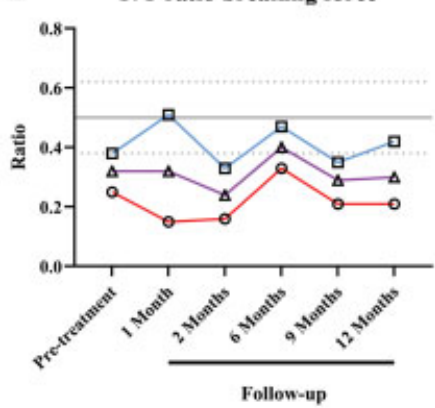

C Propulsive force

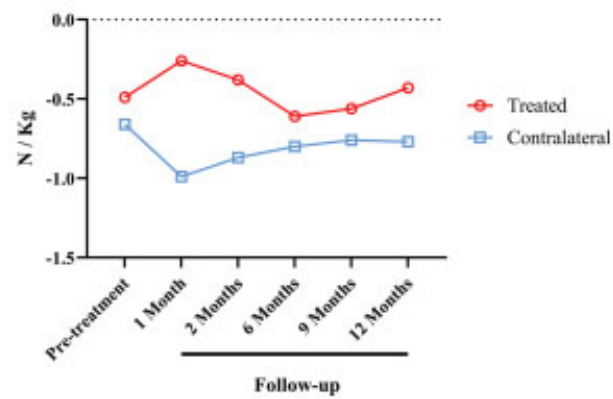

F P/T ratio propulsive force

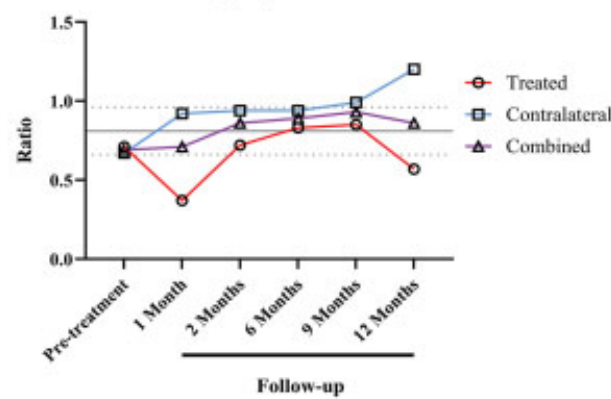

Fig. 4 Force plate analysis. Force plate analysis was performed at baseline (pre-treatment), and after 1, 2, 6, 9, and 12 months of follow-up after treatment with joint distraction. (A) Peak vertical force (Fz max), (B) breaking force (Fy +), and (C) propulsive force (Fy-) were measured in Newton $(\mathrm{N})$ and corrected for the body weight $(\mathrm{kg})$ in the treated (red line) and contralateral (blue line) hindlimbs. Furthermore, the ratio between the pelvic and thoracic limbs (P/T ratio) of the peak vertical force (D), breaking force (E), and propulsive force (F) was calculated for the treated (red line) and contralateral (blue line) limb in addition to the combined limbs (treated + contralateral [purple line]). The mean reference value (grey line) is based on a healthy population of dogs as established by Suwankong and colleagues, ${ }^{18}$ displayed with the standard variation (dotted lines).

A

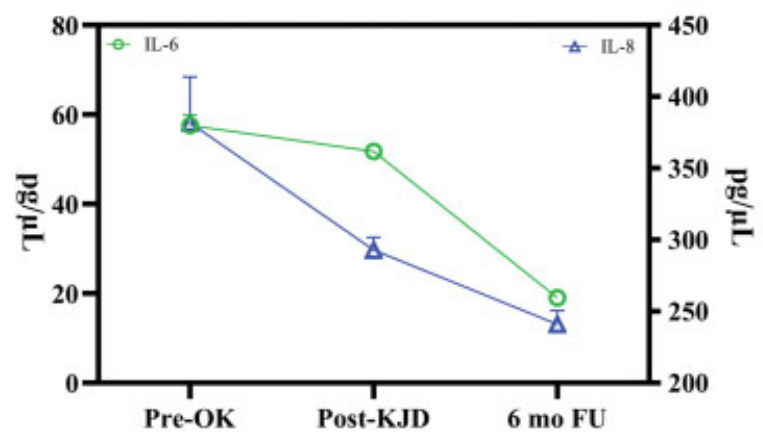

B

Cytokines

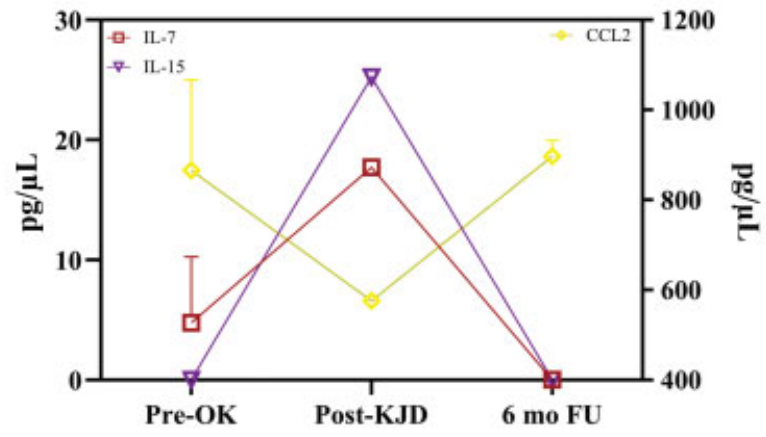

Fig. 5 Synovial fluid analysis. Cytokines were measured via a canine-specific multiplex enzyme-linked immunosorbent assay in the synovial fluid collected before the distraction treatment (pre-OK), directly after distraction treatment (post-knee joint distraction [KJD]), and after 6 months of follow-up after distraction treatment ( 6 months follow-up). All cytokines were measured in duplicate and are shown as the mean \pm standard deviation in picograms per $\mu \mathrm{L}$ (pg/ $\mathrm{LL})$. Interleukin (IL-6) (green), -7 (red), and -15 (purple) are displayed using the left Y-axis, while IL-8 (blue) and CCL2 (yellow) use the right Y-axis.

(A) Interleukin-6 and IL-8 decreased over time, while (B) IL-7 and IL-15 were increased immediately after KJD, and CCL2 changes in the opposite direction during the study period.

controlled with regular disinfection of the pin tracts and antibiotic treatment. As such, preventive measures such as adequate disinfection of the pin sites, prophylactic antibiotic medications and an early recognition of the signs could help in dogs treated with KJD. ${ }^{24}$

Another important aspect is the distraction frame strength pertaining to the weight and strength of the dog. The current KJD frame was designed based on the stature of the mixed breed dogs $(20-30 \mathrm{~kg})$ used in the study of Wiegant and colleagues. ${ }^{13}$ It is a type II (bilateral/uniplanar) external fixator and considered as a very stable fixation. However, to stimulate SF changes within the joint, the frame contains hinges that cause the tibial and femoral parts to move with respect to each other. Subsequently, the frame endures not only static- but also dynamic loading, and therefore requires an inherent metal stiffness. The stiffness 
or strength of this frame requires further attention, since a metal crack was discovered when removing the frame. Whether this was a production fault or failure of the frame is not known. Nonetheless, for larger dogs ( $>40 \mathrm{~kg}$ ) it makes sense to use thicker or stiffer frames, of stronger materials, for example, carbon fibers. ${ }^{25}$ Regarding the bone pins, an adaptation was made in this study as more (8 instead of 6 ) and thicker bone pins ( $4 \mathrm{~mm}$ instead of $3 \mathrm{~mm}$ ) were used compared with the earlier study ${ }^{13}$ to correct for the larger size of the dog. The increase in pin diameter strengthens the construct to the fourth power, thereby reducing the stress at the bone-pin interface. ${ }^{25,26}$ This adaptation prevented failure of the bone pins, a complication occasionally reported in the experimental canine studies (personal communication $\mathrm{SCM})$.

Regarding efficacy, persistent clinical improvement was found as a result of KJD, based on clinical examination and owner questionnaires. Furthermore, force plate analysis showed that there was an improvement at 6 months in the PVF compared with baseline. As with the majority of canine patients suffering from stifle $\mathrm{OA}$, this dog had radiographic $\mathrm{OA}$ in both stifle joints. Therefore, the increase in the $\mathrm{P} / \mathrm{T}$ ratio, the PVF and the PPF toward the reference values indicates the improvement in the hindlimb loading in general. At the 12-month follow-up, a small decline could be seen for the P/T ratio of the PVF and PPF. In human studies, the observed prolonged clinical effect varied in time. ${ }^{11}$ Whether this is the end of the beneficial effects of KJD in dogs or a variation in time, should be investigated in further clinical studies with longer follow-up.

Furthermore, at the beginning of this follow-up period, oral carprofen was administered and discontinued at 2 months, which might have interfered with the assessment of clinical improvement of KJD alone. However, as this report focused on the feasibility of KJD in dogs, the choice was made prioritizing the comfort of the dog. In future studies, the nonsteroidal anti-inflammatory drug administration should be discontinued (or at least protocolized) during KJD to determine the clinical effect of KJD alone. In addition, a more extensive questionnaire, such as the Liverpool Osteoarthritis in Dogs score, could be used to gather more information about the owners perspective of mobility.

To describe effects of KJD on the inflammatory processes mediating OA, OA SF biomarkers were longitudinally analysed. Interleukin- 6 and CCL2 SF levels have been reported to increase after 6 weeks of KJD in humans, although with substantial variation. ${ }^{27}$ However, in SF-derived mesenchymal stromal cells, CCL2 gene expression decreased after 3 and 6 weeks of KJD, compared with baseline. ${ }^{28}$ In dogs, increased levels IL-6, IL-8 and CCL2 have been associated with OA pain, although no correlation with radiographic OA severity or gait asymmetry was found. ${ }^{29-31}$ The increase in inflammatory cytokines IL-7 and IL-15 during KJD in this study could be explained by increased catabolic activity during KJD, as is found during joint immobilization and midway KJD in experimental dogs. ${ }^{32}$ However, in line with the clinical improvement observed, SF levels of IL-6, IL-8 and CCL2 decreased after KJD treatment. This is the first case report on the effect of KJD on canine SF biomarkers; more cases should be investigated for further interpretation.

\section{Conclusion}

This is the first case report showing that KJD was a safe and feasible treatment in a dog with severe OA. However, as only one patient was treated, clinical effectiveness should be validated in a larger cohort, preferable with a longer period of follow-up.

\section{Conflict of Interest}

None.

\section{References}

1 Anderson KL, O'Neill DG, Brodbelt DC, et al. Prevalence, duration and risk factors for appendicular osteoarthritis in a UK dog population under primary veterinary care. Sci Rep 2018;8(01): 5641

2 Allen MJ. Advances in total joint replacement in small animals. J Small Anim Pract 2012;53(09):495-506

3 Liska WD, Doyle ND. Canine total knee replacement: surgical technique and one-year outcome. Vet Surg 2009;38(05):568-582

4 Takahashi T, Baboolal TG, Lamb J, Hamilton TW, Pandit HG. Is knee joint distraction a viable treatment option for knee OA? - a literature review and meta-analysis. J Knee Surg 2019;32(08): 788-795

5 Goh EL, Lou WCN, Chidambaram S, Ma S. The role of joint distraction in the treatment of knee osteoarthritis: a systematic review and quantitative analysis. Orthop Res Rev 2019;11:79-92

6 Marijnissen ACA, van Roermund PM, van Melkebeek J, Lafeber FP. Clinical benefit of joint distraction in the treatment of ankle osteoarthritis. Foot Ankle Clin 2003;8(02):335-346

7 Paley D, Lamm BM. Ankle joint distraction. Foot Ankle Clin 2005; 10(04):685-698, ix

8 Wiegant K, van Roermund PM, Intema F, et al. Sustained clinical and structural benefit after joint distraction in the treatment of severe knee osteoarthritis. Osteoarthritis Cartilage 2013;21(11): 1660-1667

9 Intema F, Van Roermund PM, Marijnissen ACA, et al. Tissue structure modification in knee osteoarthritis by use of joint distraction: an open 1-year pilot study. Ann Rheum Dis 2011; 70(08):1441-1446

10 van der Woude JTAD, Wiegant K, van Roermund PM, et al. Fiveyear follow-up of knee joint distraction: clinical benefit and cartilaginous tissue repair in an open uncontrolled prospective study. Cartilage 2017;8(03):263-271

11 Jansen MP, van der Weiden GS, Van Roermund PM, Custers RJH, Mastbergen SC, Lafeber FPJG. Initial tissue repair predicts long-term clinical success of knee joint distraction as treatment for knee osteoarthritis. Osteoarthritis Cartilage 2018;26(12):1604-1608

12 Mastbergen SC, Marijnissen AC, Vianen ME, van Roermund PM, Bijlsma JW, Lafeber FP. The canine 'groove' model of osteoarthritis is more than simply the expression of surgically applied damage. Osteoarthritis Cartilage 2006;14(01):39-46

13 Wiegant K, Intema F, van Roermund PM, et al. Evidence of cartilage repair by joint distraction in a canine model of osteoarthritis. Arthritis Rheumatol 2015;67(02):465-474

14 Mastbergen SC, Saris DBF, Lafeber FPJG. Functional articular cartilage repair: here, near, or is the best approach not yet clear? Nat Rev Rheumatol 2013;9(05):277-290

15 Rijnberk A, van Sluijs FJ. Medical History and Physical Examination in Companion Animals. 2nd edition. Edinburgh: Saunders Ltd.; 2008:167-171 
16 Morgan JP, Voss K, Damur DM, Guerrero T, Haessig M, Montavon PM. Correlation of radiographic changes after tibial tuberosity advancement in dogs with cranial cruciate-deficient stifles with functional outcome. Vet Surg 2010;39(04):425-432

17 Brown DC, Boston RC, Coyne JC, Farrar JT. Development and psychometric testing of an instrument designed to measure chronic pain in dogs with osteoarthritis. Am J Vet Res 2007;68(06):631-637

18 Suwankong N, Meij BP, Van Klaveren NJ, et al. Assessment of decompressive surgery in dogs with degenerative lumbosacral stenosis using force plate analysis and questionnaires. Vet Surg 2007;36(05):423-431

19 Vezzoni L, Vezzoni A, Boudrieau RJ. Long-term outcome of Zürich cementless total hip arthroplasty in 439 cases. Vet Surg 2015;44 (08):921-929

20 Allen MJ, Leone KA, Lamonte K, Townsend KL, Mann KA. Cemented total knee replacement in 24 dogs: surgical technique, clinical results, and complications. Vet Surg 2009;38(05):555-567

21 Canapp SO Jr. External fracture fixation. Clin Tech Small Anim Pract 2004;19(03):114-119

22 Beever LJ, Giles K, Meeson RL. Postoperative complications associated with external skeletal fixators in dogs. Vet Comp Orthop Traumatol 2018;31(02):137-143

23 Harari J. Complications of external skeletal fixation. Vet Clin North Am Small Anim Pract 1992;22(01):99-107

24 Kazmers NH, Fragomen AT, Rozbruch SR. Prevention of pin site infection in external fixation: a review of the literature. Strateg Trauma Limb Reconstr 2016;11(02):75-85
25 Moss DP, Tejwani NC. Biomechanics of external fixation: a review of the literature. Bull NYU Hosp Jt Dis 2007;65(04):294-299

26 Bible JE, Mir HR. External fixation: Principles and applications. J Am Acad Orthop Surg 2015;23(11):683-690

27 Watt FE, Hamid B, Garriga C, et al. The molecular profile of synovial fluid changes upon joint distraction and is associated with clinical response in knee osteoarthritis. Osteoarthritis Cartilage 2020;28(03):324-333

28 Sanjurjo-Rodriguez C, Altaie A, Mastbergen S, et al. Gene expression signatures of synovial fluid multipotent stromal cells in advanced knee osteoarthritis and following knee joint distraction. Front Bioeng Biotechnol 2020;8:579751

29 Kleine SA, Gogal RM, George C, Thaliath M, Budsberg SC. Elevated synovial fluid concentration of monocyte chemoattractant protein-1 and interleukin-8 in dogs with osteoarthritis of the stifle. Vet Comp Orthop Traumatol 2020;33(02):147-150

30 Allen PI, Conzemius MG, Evans RB, Kiefer K. Correlation between synovial fluid cytokine concentrations and limb function in normal dogs and in dogs with lameness from spontaneous osteoarthritis. Vet Surg 2019;48(05):770-779

31 Syx D, Tran PB, Miller RE, Malfait AM. Peripheral mechanisms contributing to osteoarthritis pain. Curr Rheumatol Rep 2018;20 (02):9

32 Teunissen M, Miranda Bedate A, Coeleveld K, et al. Enhanced extracellular matrix breakdown characterizes the early distraction phase of canine knee joint distraction. Cartilage 2021. Doi: 19476035211014595 